Canadian Studies in Population, Vol. 15 (1), 1988, pp. 25-36

\title{
A PARITY DEPENDENT MODEL FOR THE MOST RECENT BIRTH INTERVAL
}

\author{
U.P. Singh \\ University of Gorakhpur, Gorakhpur, India \\ V.K. Singh \\ Banaras Hindu University, Varanasi, India \\ and \\ O.P. Singh \\ U.P. College, Varanasi, India
}

Résumé - Un modéle qui dépend de la parité pour représenter l'interval intergénésique le plus récent. Nous présentons des modèles de probabilité pour l'interval intergénésique le plus récent (entre la dernière et l'avant dernière naissance avant l'enquête) selon des hypothèses simplifiés des facteurs qui influencent sa distribution. Le modèle est appliqué à l'interval intergénésique sans prendre en compte la parité.

Abstract - This paper presents probability models for the most recent birth interval (the interval between last and last-but-one birth prior to survey date) under some simplified assumptions about different factors affecting its distribution. Application of the model for the interval regardless of parity has been made on a set of observed data.

Key Words - birth interval, parity, conception rate, sterility 


\section{Introduction}

Problems of evaluating family planning programmes have attracted the attention of several demographers towards analysis of data on birth intervals, since efforts to control or limit births are expected to alter the distributions of these intervals. It has also been suggested that analysis of data on birth intervals might detect the effect of these efforts before they become apparent on the birth rate (Sheps et al., 1970).

The collection of birth interval data in underdeveloped countries suffers from non-sampling errors arising out of recall lapse on the part of respondents. These errors, which are sometimes serious, often vitiate the data if the duration of time elapsed since the occurrence of the event is large (Das Gupta et al., 1955). In this context, the data on most recent birth interval (MRBI), which is defined as the interval between last and second-last birth prior to the survey date, seems to be less affected by such memory biases. Thus, they will be more reliable for the analysis of fertility changes among married women compared to other closed birth intervals (Srinivasan, 1967).

A closed birth interval is considered to be generally made up of three mutually exclusive random components in the absence of defective terminations of pregnancy (fetal losses), viz., (i) a period of temporary sterility caused by the post-partum amenorrhea following the previous birth during which no conception is possible; (ii) the menstruating period, that is, the waiting time spent in the susceptible state before the occurrence of conception; and (iii) the period of gestation leading to the next birth. Except for the last component, which is generally taken to be constant equal to nine months, the other two components vary considerably with the age of the married female and with parity. However, from the point of view of family planners, a parity dependent closed birth interval (and particularly MRBI) seems more appropriate, as these components are very much affected by the desire to control or limit births which, in turn, is associated with the number of births a married woman has already attained.

The aim of this paper is to derive continuous time probability models for the MRBI of a married female who has had a specific duration of her married life. These models assume that the expected rate of achieving conception during the susceptible state (later on termed as conception rate) and the chance of the female being sterile following a birth are functions of parity. To avoid the complexity of the models, as a first approximation the probability of incuring a fetal loss is taken to be zero. Also the period of non-susceptibility following each conception has been assumed to be constant. First, a model is developed for the interval with specific parity (Model $M_{1}$ ); next a model 
is developed regardless of parity (Model $\mathrm{M}_{2}$ ). Application of the latter model has been shown with one set of observed data.

\section{Underlying Assumptions}

Let us consider a married female with parity $n$ who has lived for time $t^{\prime}$ since her marriage. We denote by $X_{n-1}^{n}$ the MRBI, that is, the interval between $(n-1)^{t h}$ and $n^{\text {th }}$ birth. Since this interval consists of the period of gestation leading to $n^{\text {th }}$ birth which is treated to be constant equal to nine months (or 0.75 year), it is more convenient to derive the model for the random part, $X_{n-1}$, of this interval where $X_{n-I}=X_{n-1}-0.75$. However, we shall call $X_{n-I}$ the most recent birth interval without any loss of generality, as it is only a matter of substraction of a constant from $X_{n-1}^{r}$. Accordingly, the duration of married life of the female reduces to $t=t^{\prime}-0.75$. The probability distribution of $X_{n-1}$ is derived under the following assumptions:

(a) The waiting time from marriage to first conception follows an exponential distribution

$$
f_{0}(x)=\lambda_{0} e^{-\lambda_{0} x} \quad ; x>0, \lambda_{0}>0
$$

(b) The duration between $n^{\text {th }}$ and $(n+1)^{\text {th }}$ conception follows a displaced exponential distribution

$$
\begin{aligned}
& f_{n}(x)=\lambda_{n} e^{-\lambda_{n}(x-h)} \quad ; x>h, \lambda_{n}>0 \\
& \text { for } n=1,2, \ldots
\end{aligned}
$$

The parameter $\lambda_{n}(n=0,1,2, \ldots)$ appearing in expressions (1) and (2) above is called "conception rate" associated with $(n+1)^{\text {th }}$ parity and is a measure of the expected risk of a conception with which the female proceeds for $(n+1)^{t h}$ conception. $h$ is the period of non-susceptibility associated with a conception which is the sum of the gestation period and the period of post-partum amenorrhea following the birth.

(c) Every conception results in a live birth.

(d) The female is either fecund at the time of marriage or primarily sterile, that is, incapable of conceiving throughout her life. Let $\alpha_{0}$ and $\left(I-\alpha_{0}\right)$ be the respective probabilities. Further, let $\left(l-\alpha_{n}\right),(n \geq 1)$ be the probability that the female becomes sterile or chooses to be so following the 
termination of $n^{\text {th }}$ pregnancy. This may be due either to physiological sterility following $n^{\text {th }}$ birth (known as secondary sterility) or to the adoption of one of the family planning methods of 100 per cent effectiveness, with a view to preventing further pregnancies.

(e) Waiting times for conceptions are mutually independent.

These assumptions have also been used by several authors in deriving models for either the number of births to a female or the birth intervals and have been tested in a variety of situations through empirical data (see Bhattacharya et al., 1982; Singh and Singh, 1982; Singh et al., 1974 and others).

\section{Model $M_{I}$}

Let $G_{n}(x)$ denote the distribution function of the waiting time from marriage to the $(n+1)^{\text {th }}$ conception. Under the assumptions (a), (b), (c) and (d) and assuming all $\lambda_{n}$ to be distinct, Singh et al. (1974) have shown that

$$
\begin{aligned}
& G_{n}(x)=\sum_{j=0}^{n} \prod_{k=0}^{n} \frac{\lambda_{k}}{\left(\lambda_{k}-\lambda_{j}\right)}\left\{1-e^{-\lambda_{j}(x-n h)}\right\} ; x>n h \\
& k \neq j \\
& =0 \text {, otherwise ; }
\end{aligned}
$$

with $n=0,1,2 \ldots,\left[\frac{x}{h}\right]$, where $\left[\frac{x}{h}\right]$ stands for the greatest integer not exceeding $\frac{x}{h}$. The probability of exactly $n$ births in the interval of length $x, P_{n}^{\prime}(x)$, is then given by

$$
P_{n}^{\prime}(x)=G_{n-I}(x)-G_{n}(x) \quad ; x \geq 0,
$$

with $G_{n}(x)=1$, if $n<0$.

If $F_{1}(x)$ is the distribution function of the waiting time between first and second conception, then according to assumption (b)

$$
\begin{aligned}
F_{l}(x) & =0 & & ; x<h \\
& =1-e^{-\lambda_{l}(x-h)} & & ; x>h .
\end{aligned}
$$


Now, under assumptions (a) to (d), the probability distribution of the interval between last and second-last conceptions for the female with parity $n=1$ is given by

$$
\begin{aligned}
g_{l}^{\prime}(x / t) & =\frac{f_{0}(x)\left\{1-F_{1}(t-x)\right\}}{P^{\prime}(t)} & & ; 0<x<t-h \\
& =\frac{f_{0}(x)}{P_{I}^{\prime}(t)} & & ; t-h<x<t
\end{aligned}
$$

and for the female with parity $n \geq 2$, it is given by

$$
\begin{array}{rlrl}
g_{n}^{\prime}(x / t) & =0 & & ; 0<x<h \\
& =\frac{f_{n-1}(x)\left\{G_{n-2}(t-x)-G_{n}^{*}(t-x)\right\}}{P_{n}^{\prime}(t)} & ; h<x<t-\overline{n-1} h \\
& =\frac{f_{n-1}(x) G_{n-2}(t-x)}{P_{n}^{\prime}(t)} & ; t-\overline{n-1} h<x<t-\overline{n-2} h \\
=0 & ; t-\overline{n-2} h<x<t
\end{array}
$$

where

$$
\begin{aligned}
& G_{n}^{*}(t)=\sum_{j=0}^{n} \quad \prod_{k=0}^{n} \frac{\lambda_{k}}{\left(\lambda_{k}-\lambda_{j}\right)}\left\{1-e^{-\lambda_{j}(t-\overline{n-l} h)}\right\} ; t>n-1 h \\
& j \neq(n-1) \quad k \neq j \\
& k \neq(n-1) \\
& =0 \quad ; t<\overline{n-1} h
\end{aligned}
$$

and

$$
G_{n}(t)=\int_{0}^{t} f_{n-I}(x) G_{n}^{*}(t-x) d x .
$$


Now, the female with parity $n$ is either fecund or sterile. From the assump$(n-1)$

tion (e), the probability that she is fecund is given by $\Pi \alpha_{j}$ and that she is $(n-1)$ $j=0$

not by $\Pi \alpha_{j}\left(1-\alpha_{n}\right)$. Thus with this assumption the probability of exactly $j=0$

$n$ births in time $t, P_{n}(t)$, is given by

$$
P_{n}(t)=\prod_{\substack{\mathrm{j}=0 \\ \alpha_{j}}}^{n-1} G_{n-1}(t)-\prod_{j=0} \alpha_{j} G_{n}(t) .
$$

Combining the above expressions, under the stated assumptions (a) to (e), the probability distribution of the MRBI of a female with parity one (that is, $n=1$ and with specific duration of married life $t$ years is given by

$$
\begin{aligned}
g_{I}(x / t) & =\frac{\alpha_{0} f_{0}(x)\left\{1-\alpha_{I} F_{I}(t-x)\right\}}{P_{I}(t)} & ; 0<x<t-h \\
& =\frac{\alpha_{0} f_{0}(x)}{P_{I}(t)} & ; t-h<x<t
\end{aligned}
$$

For parity $n \geq 2$, the expression becomes

$$
\begin{aligned}
& g_{n}(x / t)=0 \quad ; 0<x<h \\
& =\left\{\prod_{j=0}^{n-1} \alpha_{j}\right\} \quad \frac{f_{n-I}(x)\left\{G_{n-2}(t-x)-\alpha_{n} G_{n}^{*}(t-x)\right\}}{P_{n}(t)} ; h<x<t \overline{-\bar{n}-} h \\
& =\quad\left\{\begin{array}{l}
n-1 \\
j=0
\end{array} \quad ; t-\overline{n-1} h<x<t-\overline{n-2} h\right. \\
& =0 \quad ; t-\overline{n-2} h<x<t
\end{aligned}
$$




\section{Model $M_{2}$}

Let us now consider the length of interval between last and last-but-one conceptions, $X$, regardless of parity. The probability density function of $X$ being inflated in the interval $[X>t]$ is given by

$$
\begin{aligned}
g_{I}(x) & =\sum_{n=1}^{n_{0}} g_{n}(x / t) P_{n}(t) \\
P[X>t] & =P_{0}(t),
\end{aligned}
$$

where $n_{0}=\left[\frac{t}{h}\right]+1$ and $I$ denotes inflation.

It should be pointed out here that the above models have been derived assuming all $\lambda_{n}$ 's to be distinct; however, the case of where $\lambda_{n}$ 's and $\alpha_{n}$ 's are equal may be of interest. For example, following the assumptions of Singh et al., (1974) that $\lambda_{n}=\lambda$ and $\alpha_{n}=1$ for $n=1,2, \ldots$ such that $\lambda \neq \lambda_{0}$ and $\alpha_{0} \neq$ 1 , the model $\mathrm{M}_{2}$ reduces to

$$
\begin{aligned}
& g_{I}\left(x ; \lambda_{0}, \lambda, \alpha_{0}\right)= \alpha_{0} \lambda_{0} e^{-\lambda_{0} \mathrm{x}} \mathrm{e}^{-\lambda(t-x-h)} \quad ; 0<x<h \\
&=\alpha_{0} \lambda_{0} e^{-\lambda_{0} x} e^{-\lambda(t-x-h)}+\alpha_{0} \lambda e^{-\lambda(x-h)}\left\{1-e^{-\lambda_{0}(t-x)}\right\} \\
& ; h<x<t-h \\
&=\alpha_{0} \lambda_{0} e^{-\lambda_{0} x}+\alpha_{0} \lambda e^{-\lambda(x-h)}\left\{1-e^{-\lambda_{0}(t-x)}\right\} \\
& ; t-h<x<t \\
&P I X>t] \quad=1-\alpha_{0}+\alpha_{0} e^{-\lambda_{0} t} .
\end{aligned}
$$

Application

The models derived above may be applied in a variety of situations, both for the intervals with certain parities or for the interval regardless of parity. 
Again, different relationships between conception rates $\lambda_{n}$ can be assumed. It is remarkable that parity specific birth interval models have less applicability here, for in any survey, if females with the same duration of marriage are grouped paritywise, the number of observations is not large enough to allow the theoretical distribution to be applied in order to describe the observed data.

For application purposes, we have taken data on MRBI collected in the Demographic Survey of Varanasi (Rural), 1969-70. This survey was conducted in 1969-70 under the auspices of the Demographic Research Centre, Banaras Hindu University, India. About 2,200 households scattered in 52 villages of Varanasi Tehsil were selected using a two stage stratified random sampling procedure. The reference date of the survey was October 1969. Apart from other information, a complete birth record was taken of each married female whose age was below 50 years and whose husband was alive at the time of the survey.

As in most of the surveys, in this survey we too were unable to get a sufficient number of observations on MRBI for each parity when females of a specific duration of marriage were grouped. Hence, we have applied Model $\mathrm{M}_{2}$ as an illustration to the observed data. Table 1 shows the observed distribution on MRBI irrespective of parity for those females whose age at marriage ranged from 13 to 18 years and who have spent 10 years of their married life.

On the basis of the analysis of birth intervals, based on life table techniques similar to Sheps (1965), Singh et al., (1971) have revealed that for the females with age at marriage in the range given above, one can assume $\lambda_{n}=$ $\lambda\left(\neq \lambda_{0}\right)$ and $\alpha_{n}=I\left(\neq \alpha_{0}\right)$ for $n=1,2, \ldots$. Similarly, for the surveyed population it was observed by Singh and Bhaduri (1971) that the distribution of post-partum amenorrhea associated with births is bi-modal. They have suggested that the population of married females may be assumed to be comprised of two groups with equal proportions; one with the period of temporary sterility following a birth, $h=1$ year, and another with $h=2$ years. Thus, in the light of the above results, it is reasonable to apply the following extended form of the model $\mathrm{M}_{2}$ given by expression (14):

$$
\begin{aligned}
& g_{I}^{*}\left(x ; \lambda_{0}, \lambda, \alpha_{0}\right)=0.5 g_{I}\left(x ; \lambda_{0}, \lambda, \alpha_{0} / h=h_{1}\right)+0.5 g_{I}\left(x ; \lambda_{0}, \lambda, \alpha_{0} / h=h_{2}\right) \\
& P[X>t]=1-\alpha_{0}+\alpha_{0} e^{-\lambda_{0} t}
\end{aligned}
$$

where $h_{1}=1$ year and $h_{2}=2$ years. 
TABLE 1: OBSERVED AND EXPECTED DISTRIBUTIONS OF FEMALES ACCORDING TO THE INTERVAL BETWEEN LAST AND LAST-BUT-ONE CONCEPTION

$$
(t=9.25 \text { YEARS })
$$

Time
(in years)

(1)

(2)

(3)

$\left.\begin{array}{lrr}\hline-1 & 5 \\ 1-2 & 50 & 2.4 \\ 2-3 & 85 & 56.1 \\ 3-4 & 64 & 86.3 \\ 4-5 & 24 & 47.6 \\ 5-6 & 11 & 26.8 \\ 6-7 & 6 & 15.9 \\ 7-8 & 2 \\ 8-9.25 & 14 & 10.8 \\ >9.25 & 18 & 3.8 \\ \hline \text { Total } & & 11.3\end{array}\right)$

For the estimation of the parameters $\lambda_{0}$ and $\lambda$, we use the method of moments while the estimate of another parameter $\alpha_{0}$ is obtained by equating relative frequency of females with no conception to the theoretical probability of zero conception. The first two moments of the distribution (15) are

$$
E_{t}^{*}(X)=0.5 \sum_{i=1}^{2} E_{t}\left(X / h=h_{i}\right)
$$


and

$$
V_{t}^{*}(X)=E_{t}^{*}\left(X^{2}\right)-\left[E_{t}^{*}(X)\right]^{2}
$$

where

$$
E_{t}^{*}\left(X^{2}\right)=0.5 \sum_{i=1}^{2} E_{t}^{*}\left(X^{2} / h=h_{i}\right) .
$$
$h$ are

It is easy to observe that $E_{t}(X)$ and $E_{t}\left(X^{2}\right)$ for the model (14), for given

$$
\begin{aligned}
& E_{t}\left(X / h=h_{i}\right)=\frac{1}{1-e^{-\lambda_{0} t}}\left[h_{i}+\frac{1}{\lambda}+e^{-\lambda_{0}\left(t-h_{i}\right)}\left\{\frac{\lambda\left(t-2 h_{i}\right)-1}{\lambda-\lambda_{0}}+\frac{1}{\lambda_{0}}-\frac{2 \lambda_{0}}{\left(\lambda-\lambda_{0}\right)^{2}}\right\}\right. \\
& \left.+e^{-\lambda\left(t-h_{i}\right)}\left\{\frac{(\lambda t+1) \lambda_{0}}{\lambda\left(\lambda-\lambda_{\partial}\right)}+\frac{2 \lambda_{0}}{\left(\lambda-\lambda_{0}\right)^{2}}\right\}-e^{-\lambda_{0} t}\left(t+\frac{1}{\lambda_{0}}\right)\right] ; i=1,2 . \\
& E_{t}\left(X^{2} / h=h_{i}\right)=\frac{1}{1-e^{-\lambda d^{t}}}\left[h^{2}{ }_{i}+\frac{2 h_{i}}{\lambda}+\frac{2}{\lambda^{2}}+e^{-\lambda_{0}\left(t-h_{i}\right)}\left\{\frac{\lambda t\left(t-2 h_{i}\right)+2\left(t-h_{i}\right)}{\lambda-\lambda_{0}}\right.\right. \\
& \left.+\frac{2\left(t-h_{i}\right)}{\lambda_{0}}+\frac{2}{\lambda^{2}}-\frac{2+2 \lambda t}{\left(\lambda-\lambda_{0}\right)^{2}}\right\}+e^{-\lambda\left(t-h_{i}\right)}\left\{\frac{\lambda_{0} t^{2}}{\lambda-\lambda_{0}}+\frac{2+2 \lambda t}{\left(\lambda-\lambda_{0}\right)^{2}}\right. \\
& \left.\left.-\frac{2 t}{\lambda}-\frac{2}{\lambda^{2}}\right\}-e^{-\lambda_{0} t}\left\{t^{2}+\frac{2 t}{\lambda_{0}}+\frac{2}{\lambda_{0}^{2}}\right\}\right] ; i=1,2 .
\end{aligned}
$$

The estimates which we obtained for the given data with $t=10-0.75=$ 9.25 years are $\hat{\alpha}_{0}=0.9776, \hat{\lambda}_{0}=0.34$ and $\hat{\lambda}=0.58$. The expected frequencies are given in column 3 of the table. A comparison between observed and 
expected frequencies shows that there is a close resemblance between the two. However, to provide a measure of the degree of closeness, the usual $\chi^{2}$-test has been applied. For this, the frequencies of cells 1 and 2 and of cells 8 and 9 have been grouped together, as the expected frequencies in both the cases are less than 5; though it is a classical approach of calculating the $\chi^{2}$ statistic. The $\chi^{2}$ value shows that the proposed model seems to be a reasonable one under the stated assumptions.

\section{Conclusion}

In this paper a parity specific model has been derived for MRBI. There exist a number of distributions in the literature for interlive birth intervals which are suitable for estimating the factors that govern the fertility behavior of a female and changes among them. However, the suggested model may be taken as an advancement in this direction as it uses the data on birth interval which are most recent and thus free from memory bias. The estimates obtained through the model may be viewed, therefore, as reasonable ones. Further, it can be noticed that the model can also be used to measure the changes in primary and secondary sterilities, which is not possible with other kinds of interlive birth intervals.

\section{Acknowledgments}

The authors are grateful to the referees for their valuable suggestions.

\section{References}

Bhattacharya, B.N., D. Burman, K.K. Singh and D.C. Nath. 1982. A parity dependent model for number of births. Health and Population - Perspectives and Issues 5(4):231-243.

Das Gupta, A., R.K. Som, M. Majumdar and S.N. Mitra. 1955. Couple Fertility. The National Sample Survey No. 7. Department of Economic Affairs, New Delhi, India.

Sheps, M.C. 1965. An analysis of reproductive patterns in an American isolate. Population Studies 19:65-80. 
Sheps, M.C., J.A. Menken, J.C. Ridley and J.W. Lingner. 1970. Truncation effect in closed and open birth interval data. Journal of the American Statistical Association 65:678-693.

Singh, S.N. and T. Bhaduri. 1971. On the pattern of post-partum amenorrhea. In S.N. Singh (ed.), Proceedings of All-India Seminar on Demography and Statistics. Varanasi, India: Banaras Hindu University.

Singh, S.N. and V.K. Singh. 1982. A conception dependent model for number of births and its applications. Health and Population-Perspectives and Issues 5(2):108-123.

Singh, S.N., B.N. Bhattacharya and R.C. Yadava. 1971. On some distributions for couple fertility and their applications. In S.N. Singh (ed.), Proceedings of AllIndia Seminar on Demography and Statistics. Varanasi, India: Banaras Hindu University.

1974. A parity dependent model for number of births and its applications. Sankhya 36B:93-102.

Srinivasan, K. 1967. A probability model applicable to the study of interlive birth intervals and random segments of the same. Population Studies 21:63-70.

Received July 1985; revised April 1986. 